\title{
On Fuzzy Fixed Points and an Application to Ordinary Fuzzy Differential Equations
}

\author{
Eskandar Ameer, ${ }^{1,2}$ Hassen Aydi $\mathbb{D}^{3,4}$ and Muhammad Arshad ${ }^{1}$ \\ ${ }^{1}$ Department of Mathematics, International Islamic University, H-10, Islamabad 44000, Pakistan \\ ${ }^{2}$ Department of Mathematics, Taiz University, Taiz, Yemen \\ ${ }^{3}$ Université de Sousse, Institut Supérieur d'Informatique et des Techniques de Communication, H. Sousse 4000, Tunisia \\ ${ }^{4}$ China Medical University Hospital, China Medical University, Taichung 40402, Taiwan
}

Correspondence should be addressed to Hassen Aydi; hassen.aydi@isima.rnu.tn

Received 25 September 2020; Revised 15 October 2020; Accepted 27 October 2020; Published 16 November 2020

Academic Editor: Mohamed Boussairi Jleli

Copyright ( $\odot 2020$ Eskandar Ameer et al. This is an open access article distributed under the Creative Commons Attribution License, which permits unrestricted use, distribution, and reproduction in any medium, provided the original work is properly cited.

The aim of this paper is to obtain the common fuzz fixed points of $\alpha$-fuzzy mappings satisfying generalized almost $(Y, \Lambda)$ -contraction in complete metric spaces. Our results are extensions and improvements of the several well-known recent and classical results in literature. We give an example for supporting these results. As an application, we apply our obtained results to study the existence of a solution for a second order nonlinear boundary value problem.

\section{Introduction and Preliminaries}

The fixed point result of Banach [1] is an interesting tool that ensures the existence and uniqueness of a fixed point of selfmappings defined on metric spaces. Later, various extensions and generalizations of Banach's theorem appeared by defining a variety of contractive type conditions for self and non-self-mappings on different spaces (see [2-27]).

In $[17,18]$, Berinde discussed many contraction type mappings. He initiated the notion of almost contractions.

Definition 1. A self-mapping $\widehat{S}$ on a metric space (in short MS) $(\omega, \partial)$ is named as an almost contraction, if there are $\lambda \in[0,1)$ and $L \geq 0$ so that for all $r, \widehat{J} \in \omega$,

$$
\partial(\widehat{S}(r), \widehat{S}(\widehat{J}))<\lambda \partial(r, \widehat{j})+L \partial(\hat{j}, \widehat{S}(\hat{j})) .
$$

This almost contraction has been generalized as follows.

Definition 2. A self-mapping $\widehat{S}$ on a MS $(\omega, \partial)$ is called a generalized almost contraction, if there are $\lambda \in[0,1)$ and $L$ $\geq 0$ so that for all $r, \widehat{J} \in \omega$,

$$
\partial(\widehat{S}(r), \widehat{S}(\widehat{J}))<\lambda \partial(r, \widehat{J})+L \min \left\{\partial(v, \widehat{S} v), \partial\left(\iota, \widehat{S}_{\iota}\right), \partial\left(v, \widehat{S}_{\iota}\right), \partial(\iota, \widehat{S} v)\right\}
$$

Wardowski [28] presented the notion of F-contractions and established a related fixed point theorem.

Definition 3 (see [28]). A self-mapping $\widehat{S}$ on a MS $(\omega, \partial)$ said to be an $F$-contraction if there are $F \in F$ and $\tau>0$ such that

$$
\begin{aligned}
\forall r, \widehat{J} \in \omega, \partial(\widehat{S}(r), \widehat{S}(\widehat{J})) & >0 \Rightarrow \tau+F(\partial(\widehat{S}(r), \widehat{S}(\widehat{J}))) \\
& \leq F(\partial(r, \widehat{J}))
\end{aligned}
$$

where $F$ is the set of functions $F:(0, \infty) \longrightarrow(-\infty, \infty)$ such that

(F1) For all $0<r<\widehat{J}, F(r)<F(\widehat{J})$,

(F2) For each sequence $\left\{t_{n}\right\}_{n=1}^{\infty}$ of positive numbers,

$$
\lim _{n \rightarrow \infty} F(t)=-\infty \text { ifandonlyif } \lim _{n \rightarrow \infty} t_{n}=0
$$

(F3) There is $k \in(0,1)$ so that $\lim _{t \rightarrow 0^{+}} t^{k} F(t)=0$,

Later on, Altun et al. [22] modified Definition 3 by adding the condition (F4): 
(F4) $F(\inf (P))=\inf (F(P))$, for all $P \subset(0, \infty)$ with inf $(P)>0$.

Denote by $F^{*}$ the set of functions $F:(0, \infty) \longrightarrow(-\infty, \infty)$ verifying the conditions $(F 1)-(F 4)$.

Theorem 4 (see [28]). Let $\widehat{S}: \omega \longrightarrow \omega$ be an $F$-contraction on a complete MS. Then, $T$ admits a unique fixed point $s^{*} \in \omega$, and for each $r \in \omega$, the sequence $\left\{T^{j} r\right\}_{j \in \mathbb{N}}$ is convergent to $r^{*}$.

Definition 5 (see [4]). A self-mapping $\widehat{S}$ on a MS $(\omega, \partial)$ is said to be a $\theta$-contraction, if there are $\ell \in(0,1)$ and $\theta \in \Theta$ so that

$r, \widehat{J} \in \omega, \partial(\widehat{S}(r), \widehat{S}(\widehat{J})) \neq 0 \Rightarrow \theta(\partial(\widehat{S}(r), \widehat{S}(\widehat{J}))) \leq[\theta(\partial(r, J \wedge))]^{\ell}$

where $\Theta$ is the set of functions $\theta:(0, \infty) \longrightarrow(1, \infty)$ so that

$(\Theta 1) \theta$ is nondecreasing,

$(\Theta 2)$ For each sequence $\left\{t_{n}\right\} \subset(0, \infty)$,

$$
\lim _{n \rightarrow \infty} \theta\left(t_{n}\right)=1 \text { if and only if } \lim _{n \rightarrow \infty} t_{n}=0^{+},
$$

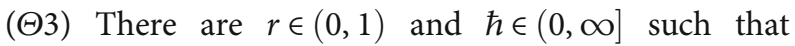
$\lim _{t \rightarrow 0^{+}}\left(\theta(t)-1 / t^{r}\right)=\hbar$,

$(\Theta 4) \theta$ is continuous. [4].

The following result was established by Jleli and Samet

Theorem 6 (see [4]). Each $\theta$-contraction mapping on a complete MS $(\omega, \partial)$ possesses a unique fixed point.

As in [22], Hancer et al. [12] took the following: $Q>0$.

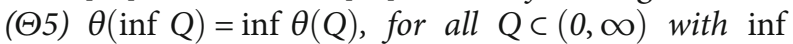

Denote by $\Theta^{*}$ the set of functions $Q:(0, \infty) \longrightarrow(1, \infty)$ so that $(\Theta 1),(\Theta 2),(\Theta 3)$, and $(\Theta 5)$ hold.

As in [9], we denote by $\Xi$ the set of functions $\theta:(0, \infty)$ $\longrightarrow(1, \infty)$ so that

$(\Theta 1)^{\prime} \theta$ is nondecreasing,

$(\Theta 2)^{\prime} \inf _{t \in(0, \infty)} \theta(t)=1$,

$(\Theta 3)^{\prime} \theta$ is continuous.

Theorem 7 (see [9]). Let $\widehat{S}$ be a self-mapping on a $M S(\omega, \partial)$. We have the equivalence of the following:

(i) $\widehat{S}$ is a $\theta$-contraction with $\theta \in \Xi$;

(ii) $\widehat{S}$ is a F-contraction with $F \in F$.

Very recently, Liu et al. [9] introduced the notion of $(Y, \Lambda$ )-type contractions and established new fixed point theorems for such kind of mappings in complete MS.

Definition 8. A self-mapping $\widehat{S}$ on a $\operatorname{MS}(\omega, \partial)$ is said to be a $(Y, \Lambda)$-type contraction, if there are a comparison function
$Y$ and $\phi \in \Phi$ such that for all $r, \widehat{J} \in \omega$,

$$
\partial(\widehat{S}(r), \widehat{S}(\widehat{J}))>0 \Rightarrow \Lambda(\partial(\widehat{S}(r), \widehat{S}(\widehat{J}))) \leq Y[\Lambda(M(r, \widehat{J}))]
$$

where

$M(r, \widehat{J})=\max \left\{\partial(r, \widehat{J}), \partial(r, \widehat{S}(r)), \partial(\widehat{J}, \widehat{S}(\widehat{J})), \frac{\partial(r, \widehat{S}(\widehat{J}))+\partial(\widehat{J}, \widehat{S}(r))}{2}\right\}$

and $\Phi$ is the set of functions $\Lambda:(0, \infty) \longrightarrow(0, \infty)$ so that

(Ф1) $\Lambda$ is nondecreasing,

$(\Phi 2)$ For each sequence $\left\{t_{n}\right\} \subset(0, \infty)$,

$$
\lim _{n \rightarrow \infty} \Lambda\left(t_{n}\right)=0 \text { iff } \lim _{n \rightarrow \infty} t_{n}=0
$$

(Ф3) $\Lambda$ is continuous

As in [2], a function $Y:(0, \infty) \longrightarrow(0, \infty)$ is named as a comparison function if

(1) $Y$ is increasing, that is, $t_{1}<t_{2} \Rightarrow Y\left(t_{1}\right) \leq Y\left(t_{2}\right)$,

(2) $\lim _{n \rightarrow \infty}^{n} Y(t)=0$ for all $t>0$, where $Y^{n}$ stands for the nth iterate of $Y$.

Denote by $\Psi$ the set of comparison functions.

Lemma 9 (see [9]). Let $\Lambda:(0, \infty) \longrightarrow(0, \infty)$ be a nondecreasing and continuous function with $\inf _{t \in(0, \infty)} \Lambda(t)=0$, and $\left\{t_{k}\right\}_{k}$ be a sequence in $(0, \infty)$. Then,

$$
\lim _{k \rightarrow \infty} \Lambda\left(t_{k}\right)=0 \quad \text { iff } \lim _{k \rightarrow \infty} t_{k}=0
$$

Example 10 (see [2]). Consider the following comparison functions:

(1) $Y(t)=\lambda t$ with $0<\lambda<1$,

$$
\begin{gathered}
Y(t)= \begin{cases}\frac{t}{2}, & 0<t<1, \\
\frac{t}{3}, & 1 \leq t,\end{cases} \\
Y(t)=\frac{t}{t+1} .
\end{gathered}
$$

Example 11 (see [9]). For all $t \in(0, \infty)$, consider

$$
\begin{gathered}
\Lambda_{1}(\eta)=t \\
\Lambda_{2}(\eta)=\sqrt{t \sqrt{t}} \\
\Lambda_{3}(\eta)=t e^{t}
\end{gathered}
$$

Here, $\Lambda_{1}, \Lambda_{2}, \Lambda_{3} \in \Phi$. 
On the other hand, using the notion of a fuzzy set, Heilpern [29] initiated the family of fuzzy mappings, which generalized the concept of set-valued mappings, and presented a fixed point result for fuzzy contraction mappings in the context of metric linear spaces. Mention that the theorem of Heilpern [29] is considered as a fuzzy extension of the BCP. Later on, several researchers worked on fixed point results involving fuzzy mappings, see ([30-46]).

Let $C B(\omega)$ be the set family of bounded and closed subsets of a MS $(\omega, \partial)$. For $r \in \omega$ and $A, B \in C B(\omega)$, set

$$
\partial(r, B)=\inf _{\widehat{J} \in B} \partial(r, \widehat{J}) .
$$

Given $H: C B(\omega) \times C B(\omega) \longrightarrow[0,1)$ as

$$
H(A, B)=\max \left\{\sup _{r \in A} \partial(r, B), \sup _{\widehat{J} \in B} \partial(\widehat{J}, A)\right\} .
$$

$H$ is a metric on $C B(\omega)$. It is named as the PompeiuHausdorff metric induced by $\partial$. A fuzzy set in $\omega$ is a function with domain $\omega$ and values in $[0,1]$. Denote by $I^{\omega}$ the set fuzzy sets in $\omega$. Let $A$ be a fuzzy set and $r \in \omega$, then the function values $A(r)$ is named as the grade of membership of $r$ in $A$. The $\alpha$-level set of $A$ is denoted by $[A]_{\alpha}$ and is defined by

$$
[A]_{\alpha}=\{\omega: \Lambda(r) \geq \alpha\} \text { if } \alpha \in(0,1] \text { and }[A]_{0}=\{\omega: \Lambda \overline{(r)}>0\} .
$$

Here, $\bar{B}$ denotes the closure of the set $B$. Let $\mathfrak{I}(\omega)$ be the collection of all fuzzy sets in $\omega$. For $A, B \in \mathfrak{I}(\omega), A \subset B$ means $A(r) \leq B(r)$ for each $r \in \omega$. The fuzzy set $\chi_{\{r\}}$ is denoted by $\{r\}$ unless and until it is stated, where $\chi_{\{r\}}$ is the characteristic function of the crisp set $A$. If there is $\alpha \in[0,1]$ so that $[A]_{\alpha},[B]_{\alpha} \in C B(\omega)$, then consider

$$
P_{\alpha}(A, B)=\inf _{r \in[A]_{\alpha}, \widehat{J} \in[B]_{\alpha}} \partial(r, \widehat{J}), D_{\alpha}(A, B)=H\left([A]_{\alpha},[B]_{\alpha}\right) .
$$

$$
\text { If }[A]_{\alpha},[B]_{\alpha} \in C B(\omega) \text { for each } \alpha \in[0,1] \text {, then take }
$$

$$
P(A, B)=\sup _{\alpha} P_{\alpha}(A, B), \partial_{\infty}(A, B)=\sup _{\alpha} D_{\alpha}(A, B) .
$$

We write $p(r, B)$ instead of $p(\{r\}, B)$. A fuzzy set $A$ in a metric linear space $V$ is said to be an approximate quantity if and only if $[A]_{\alpha}$ is compact and convex in $V$ for each $\alpha$ $\epsilon[0,1]$ and $\sup _{r \in V} A(r)=1$. The set of approximate quantities in $V$ is denoted by $W(V)$. Let $\omega$ be an arbitrary set and $Y$ be a MS. A mapping $\widehat{S}$ is called fuzzy mapping (in short, FM) if $\widehat{S}$ is a mapping from $\omega$ into $\mathfrak{J}(Y)$. A fuzzy mapping $\widehat{S}$ is a fuzzy subset on $\omega \times Y$ with the membership function $\widehat{S}(r)(\widehat{J})$. The function $\widehat{S}(r)(\widehat{J})$ is the grade of membership of $\widehat{J}$ in $\widehat{S}(r)$.

Definition 12. Let $\widehat{S}, \Gamma$ be $F M$ from $\omega$ into $\mathfrak{I}(Y)$. An element $\sigma$ in $\omega$ is said to be an $\alpha$-fuzzy fixed point of $\Gamma$ if there is $\alpha$ $\epsilon[0,1]$ so that $\sigma \in[\Gamma u]_{\alpha} . \sigma \in \omega$ is named as a common $\alpha$ -fuzzy fixed point of $\widehat{S}$ and $\Gamma$ if there is $\alpha \in[0,1]$ so that $\sigma$ $\in[\widehat{S} u]_{\alpha} \cap[\Gamma \sigma]_{\alpha} . \alpha=1$ is called a common fixed point of fuzzy mappings.

In this manuscript, we ensure the existence of some common $\alpha$-fuzzy fixed points for fuzzy mappings for almost $(Y, \Lambda)$-contractions in the class of complete MS. Our theorems generalize some known results in literature.

Now, we need the following.

Lemma 13 (see [16]). Let ( $\omega, \partial)$ be a MS and $A, B \in C B(\omega)$, then for every $\eta \in A$,

$$
\partial(\eta, B) \leq H(A, B) .
$$

Lemma 14 (see [30]). Let $V$ be a metric linear space and $\widehat{S}$ $: V \longrightarrow W(V)$ be a fuzzy mapping and $\rho_{0} \in V$. Then, there is $\rho_{1} \in V$ so that $\left\{\rho_{1}\right\} \subset \widehat{S}\left(\rho_{0}\right)$.

Lemma 15 (see [47]). Let $(\omega, \partial)$ be a metric space, $r^{*} \in \omega$ and $\widehat{\mathcal{S}}, \Gamma: \omega \longrightarrow \mathfrak{I}(\omega)$ be FM such that $\widehat{S}(r)$ is a nonempty compact set for each $r \in \omega$. Then, $r^{*} \in \widehat{S}\left(r^{*}\right)$ if and only if $\widehat{S}\left(r^{*}\right)\left(r^{*}\right) \geq \widehat{S}\left(r^{*}\right)(r)$ for each $r \in \omega$.

\section{Main Results}

From now on, $(\omega, \partial)$ is assumed to be a complete MS.

Theorem 16. Let $\widehat{S}, \Gamma: \omega \longrightarrow \mathfrak{I}(\omega)$ be FM and for each $r \in \omega$, and there exist $\alpha_{\widehat{S}}(r), \alpha_{\Gamma}(r) \in(0,1]$ such that $[\widehat{S}(r)]_{\alpha_{\hat{S}}(r)}$ and $[\Gamma(\widehat{J})]_{\alpha_{T}(r)}$ are nonempty, closed, and bounded subsets of $\omega$. Assume that there exist $Y \in \Psi, \Lambda \in \Phi$, and $L \geq 0$ such that for all $\left.r, \widehat{J} \in \omega, H(\widehat{S}(r)]_{\alpha_{\widehat{S}}(r)},[\Gamma(\widehat{J})]_{\alpha_{\Gamma}(\widehat{J})}\right)>0$ implies

$$
\Lambda\left(H\left([\widehat{S}(r)]_{\alpha_{\widehat{S}}(r)^{\prime}},[\Gamma(\widehat{J})]_{\alpha_{\Gamma}(\widehat{J})}\right)\right) \leq Y(\Lambda(M(r, \widehat{J})))+L E(r, \widehat{J}),
$$

where

$$
M(r, \widehat{J})=\max \left\{\begin{array}{c}
\partial(r, \widehat{J}), \partial\left(r,[\widehat{S}(r)]_{\alpha_{S}(r)}\right), \\
\partial\left(\hat{J},[\Gamma(\widehat{J})]_{\alpha_{T}(\hat{J})}\right), \frac{\partial\left(r,[\Gamma(\widehat{J})]_{\alpha_{r}(\hat{J})}\right)+\partial\left(\hat{J},[\widehat{S}(r)]_{\alpha_{\widehat{S}}(r)}\right)}{2}
\end{array}\right\},
$$

$$
E(r, \widehat{J})=\min \left\{\begin{array}{c}
\partial\left(r,[\widehat{S}(r)]_{\alpha_{\widehat{S}}(r)}\right), \partial\left(\widehat{J},[\Gamma(\widehat{J})]_{\alpha_{T}(\widehat{J})}\right) \\
, \partial\left(r,[\Gamma(\widehat{J})]_{\alpha_{T}(\widehat{J})}\right), \partial\left(\widehat{J},[\widehat{S}(r)]_{\alpha_{\widehat{S}(r)}}\right)
\end{array}\right\} .
$$

If $Y$ is continuous, then there exists some $u \in \omega$ such that $u \in[\widehat{S}(u)]_{\alpha_{\widehat{S}}(u)} \cap[\Gamma(u)]_{\alpha_{\Gamma}(u)}$. 
Proof. Let $r_{0} \in \omega$. By hypotheses, there exists $\alpha_{\widehat{S}}\left(r_{0}\right) \in(0,1]$ such that $\left[\widehat{S}\left(r_{0}\right)\right]_{\alpha_{\hat{S}}\left(r_{0}\right)}$ is a nonempty, closed, and bounded subset of $\omega$. For convenience, we denote $\alpha_{\widehat{S}}\left(r_{0}\right)$ by $\alpha_{1}$. Let $r_{1}$ $\in\left[\widehat{S}\left(r_{0}\right)\right]_{\alpha_{\widehat{S}}\left(x_{0}\right)}$, then there exists $\alpha_{\Gamma}\left(r_{1}\right) \in(0,1]$ such that $\left[\Gamma\left(r_{1}\right)\right]_{\alpha_{\Gamma}\left(r_{1}\right)}$ is a nonempty, closed, and bounded subset of $\omega$ . Since $\Lambda$ is nondecreasing, we have used (19) and Lemma 13

$$
\begin{aligned}
\Lambda\left(\partial\left(r_{1},\left[\Gamma\left(r_{1}\right)\right]_{\alpha_{\Gamma}\left(r_{1}\right)}\right)\right) & \leq \Lambda\left(H\left(\left[\widehat{S}\left(r_{0}\right)\right]_{\alpha_{\widehat{S}}\left(r_{0}\right)}\left[\Gamma\left(r_{1}\right)\right]_{\alpha_{\Gamma}\left(r_{1}\right)}\right)\right) \\
& \leq Y\left(\Lambda\left(M\left(r_{0}, r_{1}\right)\right)\right)+L E\left(r_{0}, r_{1}\right),
\end{aligned}
$$

where

$M\left(r_{0}, r_{1}\right)=\max \left\{\begin{array}{c}\partial\left(r_{0}, r_{1}\right), \partial\left(r_{0},\left[\widehat{S}\left(r_{0}\right)\right]_{\alpha_{\hat{S}}\left(r_{0}\right)}\right), \partial\left(r_{1},\left[\Gamma\left(r_{1}\right)\right]_{\alpha_{T}\left(r_{1}\right)}\right), \\ \frac{\partial\left(r_{0},\left[\Gamma\left(r_{1}\right)\right]_{\alpha_{\Gamma}\left(r_{1}\right)}\right)+\partial\left(r_{1},\left[\widehat{S}\left(r_{0}\right)\right]_{\alpha_{\widehat{S}}\left(r_{0}\right)}\right)}{2}\end{array}\right\}$,

$E\left(r_{0}, r_{1}\right)=\min \left\{\begin{array}{l}\partial\left(r_{0},\left[\widehat{S}\left(r_{0}\right)\right]_{\alpha_{\widehat{S}}\left(r_{0}\right)}\right), \partial\left(r_{1},\left[\Gamma\left(r_{1}\right)\right]_{\alpha_{\Gamma}\left(r_{1}\right)}\right), \\ \partial\left(r_{0},\left[\Gamma\left(r_{1}\right)\right]_{\alpha_{\Gamma}\left(r_{1}\right)}\right), \partial\left(r_{1},\left[\widehat{S}\left(r_{0}\right)\right]_{\alpha_{\widehat{S}}\left(r_{0}\right)}\right)\end{array}\right\}$.

By (Ф3), we have

$$
\Lambda\left(\partial\left(r_{1},\left[\Gamma\left(r_{1}\right)\right]_{\alpha_{\Gamma}\left(r_{1}\right)}\right)\right)=\inf _{\hat{J} \in\left[\Gamma\left(r_{1}\right)\right]_{\alpha_{\Gamma}\left(r_{1}\right)}} \Lambda\left(\partial\left(r_{1}, \widehat{J}\right)\right) .
$$

Thus, there exists $r_{1} \in\left[\Gamma\left(r_{1}\right)\right]_{\alpha_{T}\left(r_{1}\right)}$ such that

$$
\Lambda\left(\partial\left(r_{1},\left[\Gamma\left(r_{1}\right)\right]_{\alpha_{\Gamma}\left(r_{1}\right)}\right)\right)=\Lambda\left(\partial\left(r_{1}, r_{2}\right)\right) .
$$

Then from (22), we have

$$
\Lambda\left(\partial\left(r_{1}, r_{2}\right)\right) \leq Y\left(\Lambda\left(M\left(r_{0}, r_{1}\right)\right)\right)+L E\left(r_{0}, r_{1}\right),
$$

where

$$
\begin{aligned}
M\left(r_{0}, r_{1}\right) & =\max \left\{\begin{array}{c}
\partial\left(r_{0}, r_{1}\right), \partial\left(r_{0}, r_{1}\right), \partial\left(r_{1}, r_{2}\right), \\
\frac{\partial\left(r_{0}, r_{2}\right)+\partial\left(r_{1}, r_{1}\right)}{2}
\end{array}\right\} \\
& =\max \left\{\partial\left(r_{0}, r_{1}\right), \partial\left(r_{1}, r_{2}\right)\right\}, \\
E\left(r_{0}, r_{1}\right) & =\min \left\{\begin{array}{l}
\partial\left(r_{0}, r_{1}\right), \partial\left(r_{1}, r_{2}\right), \\
\partial\left(x_{0}, x_{2}\right), \partial\left(r_{1}, r_{1}\right)
\end{array}\right\}=0 .
\end{aligned}
$$
have

If $\max \left\{\partial\left(r_{0}, r_{1}\right),\left(r_{1}, r_{2}\right)\right\}=\partial\left(r_{1}, r_{2}\right)$, then from (27), we

$$
\Lambda\left(\partial\left(r_{1}, r_{2}\right)\right) \leq Y\left(\Lambda\left(\partial\left(r_{1}, r_{2}\right)\right)\right)<\Lambda\left(\partial\left(r_{1}, r_{2}\right)\right),
$$

which is a contradiction. Thus, $\max \left\{\partial\left(r_{0}, r_{1}\right),\left(r_{1}, r_{2}\right)\right\}=$ $\partial\left(r_{0}, r_{1}\right)$. By (27), we get that

$$
\Lambda\left(\partial\left(r_{1}, r_{2}\right)\right) \leq Y\left(\Lambda\left(\partial\left(r_{0}, r_{1}\right)\right)\right) .
$$

Next, there exists $\alpha_{\widehat{S}}\left(r_{2}\right) \in(0,1]$ such that $\left[\widehat{S}\left(r_{2}\right)\right]_{\alpha_{\widehat{S}}\left(r_{2}\right)}$ is a nonempty, closed, and bounded subset of $\omega$. By Lemma 13, using (19) and the fact that $\Lambda$ is nondecreasing, we have

$$
\begin{aligned}
\Lambda\left(\partial\left(r_{2},\left[\widehat{S}\left(r_{2}\right)\right]_{\alpha_{\hat{S}}\left(r_{2}\right)}\right)\right) & \leq \Lambda\left(H\left(\left[\widehat{S}\left(r_{2}\right)\right]_{\alpha_{\widehat{S}}\left(r_{2}\right)},\left[\Gamma\left(r_{1}\right)\right]_{\alpha_{\Gamma}\left(r_{1}\right)}\right)\right) \\
& \leq Y\left(\Lambda\left(M\left(r_{1}, r_{2}\right)\right)\right)+L E\left(r_{1}, r_{2}\right),
\end{aligned}
$$

where

$M\left(r_{1}, r_{2}\right)=\max \left\{\begin{array}{c}\partial\left(r_{1}, r_{2}\right), \partial\left(r_{2},\left[\widehat{S}\left(r_{2}\right)\right]_{\alpha_{\hat{S}}\left(r_{2}\right)}\right), \partial\left(r_{1},\left[\Gamma\left(r_{1}\right)\right]_{\alpha_{\Gamma}\left(r_{1}\right)}\right), \\ \frac{\partial\left(r_{2},\left[\Gamma\left(r_{1}\right)\right]_{\alpha_{\Gamma}\left(r_{1}\right)}\right)+\partial\left(r_{1},\left[\widehat{S}\left(r_{2}\right)\right]_{\alpha_{\tilde{S}}\left(r_{2}\right)}\right)}{2}\end{array}\right\}$,

$E\left(r_{1}, r_{2}\right)=\min \left\{\begin{array}{l}\partial\left(r_{2},\left[\widehat{S}\left(r_{2}\right)\right]_{\alpha_{\widehat{S}}\left(r_{2}\right)}\right), \partial\left(r_{1},\left[\Gamma\left(r_{1}\right)\right]_{\alpha_{\Gamma}\left(r_{1}\right)}\right), \\ \partial\left(r_{2},\left[\Gamma\left(r_{1}\right)\right]_{\alpha_{\Gamma}\left(r_{1}\right)}\right), \partial\left(r_{1},\left[\widehat{S}\left(r_{2}\right)\right]_{\alpha_{\widehat{S}}\left(r_{2}\right)}\right)\end{array}\right\}$.

From $(\Phi 4)$, we have

$$
\Lambda\left(\partial\left(r_{2},\left[\widehat{S}\left(r_{2}\right)\right]_{\alpha_{\widehat{S}}\left(r_{2}\right)}\right)\right)=\inf _{\widehat{J}_{1} \in\left[\widehat{S}\left(r_{2}\right)\right]_{\alpha_{\widehat{S}}\left(r_{2}\right)}} \Lambda\left(\partial\left(r_{2}, \widehat{J}_{1}\right)\right) .
$$

Thus, there exists $r_{3} \in\left[\widehat{S}\left(r_{2}\right)\right]_{\alpha_{\hat{S}}\left(r_{2}\right)}$ such that

$$
\Lambda\left(\partial\left(r_{2},\left[\widehat{S}\left(r_{2}\right)\right]_{\alpha_{\hat{S}}\left(r_{2}\right)}\right)\right)=\Lambda\left(\partial\left(r_{2}, r_{3}\right)\right) .
$$

Then from (32), we have

$$
\Lambda\left(\partial\left(r_{2}, r_{3}\right)\right) \leq Y\left(\Lambda\left(M\left(r_{1}, r_{2}\right)\right)\right)+L E\left(r_{1}, r_{2}\right),
$$

where

$$
\begin{aligned}
M\left(r_{1}, r_{2}\right) & =\max \left\{\begin{array}{c}
\partial\left(r_{1}, r_{2}\right), \partial\left(r_{2}, r_{3}\right), d\left(r_{1}, r_{2}\right), \\
\frac{\partial\left(r_{1}, r 3\right)+\partial\left(r_{2}, r_{2}\right)}{2}
\end{array}\right\} \\
& =\max \left\{\partial\left(r_{1}, r_{2}\right), \partial\left(r_{2}, r_{3}\right)\right\}, \\
E\left(r_{1}, r_{2}\right) & =\min \left\{\begin{array}{l}
\partial\left(r_{2}, r_{3}\right), \partial\left(r_{1}, r_{2}\right), \\
\partial\left(r_{2}, r_{2}\right), \partial\left(r_{1}, r_{3}\right)
\end{array}\right\}=0 .
\end{aligned}
$$

If $\max \left\{\partial\left(r_{1}, r_{2}\right),\left(r_{2}, r_{3}\right)\right\}=\partial\left(r_{2}, r_{3}\right)$, then from (37), we have

$$
\Lambda\left(\partial\left(r_{2}, r_{3}\right)\right) \leq Y\left(\Lambda\left(\partial\left(r_{2}, r_{3}\right)\right)\right)<\Lambda\left(\partial\left(r_{2}, r_{3}\right)\right),
$$


which is a contradiction. Thus, $\max \left\{\left(r_{1}, r_{2}\right),\left(r_{2}, r_{3}\right)\right\}=\partial$ $\left(r_{1}, r_{2}\right)$. By (37), we get that

$$
\Lambda\left(\partial\left(r_{2}, r_{3}\right)\right) \leq Y\left(\Lambda\left(\partial\left(r_{1}, r_{2}\right)\right)\right) .
$$

By continuing this process, we construct a sequence $\left\{r_{n}\right\}$ in $\omega$ such that $r_{2 n+1} \in\left[\widehat{S}\left(r_{2 n}\right)\right]_{\alpha_{\widehat{S}}\left(r_{2 n}\right)}$ and $r_{2 n+2} \epsilon$ $\left[\Gamma\left(r_{2 n+1}\right)\right]_{\alpha_{\Gamma}\left(r_{2 n+1}\right)}$

$\Lambda\left(\partial\left(r_{2 n+1}, r_{2 n+2}\right)\right) \leq Y\left(\Lambda\left(\partial\left(r_{2 n}, r_{2 n+1}\right)\right)\right)$, for all $n \in \mathbb{N}$.

$\Lambda\left(\partial\left(r_{2 n+2}, r_{2 n+3}\right)\right) \leq Y\left(\Lambda\left(\partial\left(r_{2 n+1}, r_{2 n+2}\right)\right)\right)$, for all $n \in \mathbb{N}$.

Thus, from (42) and (43), we have

$$
\Lambda\left(\partial\left(r_{n}, r_{n+1}\right)\right) \leq Y\left(\Lambda\left(\partial\left(r_{n-1}, r_{n}\right)\right)\right), \text { for all } n \in \mathbb{N} .
$$

This implies that

$$
\begin{aligned}
\Lambda\left(\partial\left(r_{n}, r_{n+1}\right)\right) & \leq Y\left(\Lambda\left(\partial\left(r_{n-1}, r_{n}\right)\right)\right) \leq Y^{2}\left(\Lambda\left(\partial\left(r_{n-2}, r_{n-1}\right)\right)\right) \\
& \leq \cdots \leq Y^{n}\left(\Lambda\left(\partial\left(r_{0}, r_{1}\right)\right)\right) .
\end{aligned}
$$

Letting $n \longrightarrow \infty$, we get

$0 \leq \lim _{n \rightarrow \infty} \Lambda\left(\partial\left(r_{n}, r_{n+1}\right)\right) \leq \lim _{n \rightarrow \infty} Y^{n}\left(\Lambda\left(\partial\left(r_{0}, r_{1}\right)\right)\right)=0$.

It yields that

$$
\lim _{n \rightarrow \infty} \Lambda\left(\partial\left(r_{n}, r_{n+1}\right)\right)=0 .
$$

This together with $(\Phi 2)$ and Lemma 9 gives that

$$
\lim _{n \rightarrow \infty} \partial\left(r_{n}, r_{n+1}\right)=0 .
$$

Now, we will prove that $\left\{r_{n}\right\}$ is a Cauchy sequence. Arguing by contradiction, we assume that there are $\varepsilon>$ 0 and sequences $\left\{p_{n}\right\}_{n=1}^{\infty}$ and $\left\{q_{n}\right\}_{n=1}^{\infty}$ of integers so that for all $n \in \mathbb{N}, p_{n}>q_{n}>n$ with $d\left(r_{p(n)}, r_{q(n)}\right) \geq \varepsilon$,

$$
\partial\left(r_{p(n)-1}, r_{q(n)}\right)<\varepsilon .
$$

Thus,

$$
\begin{aligned}
\varepsilon & \leq \partial\left(r_{p(n)}, r_{q(n)}\right) \leq \partial\left(r_{p(n)}, r_{p(n)-1}\right)+\partial\left(r_{p(n)-1}, r_{q(n)}\right) \\
& \leq \partial\left(r_{p(n)}, r_{p(n)-1}\right)+\varepsilon .
\end{aligned}
$$

Letting $n \longrightarrow \infty$ in (2.13), we get

$$
\lim _{n \rightarrow \infty} \partial\left(r_{p(n)}, r_{q(n)}\right)=\varepsilon .
$$

Again,

$$
\begin{aligned}
\partial\left(r_{p(n)}, r_{q(n)}\right) \leq & \partial\left(r_{p(n)}, r_{p(n)+1}\right)+\partial\left(r_{p(n)+1}, r_{q(n)+1}\right) \\
+ & \partial\left(r_{q(n)+1}, r_{q(n)}\right), \\
\partial\left(r_{p(n)+1}, r_{q(n)+1}\right) \leq & \partial\left(r_{p(n)+1}, r_{p(n)}\right)+\partial\left(r_{p(n)}, r_{q(n)}\right) \\
& +\partial\left(r_{q(n)}, r_{q(n)+1}\right) .
\end{aligned}
$$

Taking $n \longrightarrow \infty$ in (51) and (52), we get

$$
\lim _{n \rightarrow \infty} \partial\left(r_{p(n)+1}, r_{q(n)+1}\right)=\varepsilon .
$$

From (48) and (51), we can choose an integer $n_{0} \geq 1$ so that by (19), we get

$$
\begin{aligned}
& \Lambda\left(\partial\left(r_{p(n)+1}, r_{q(n)+1}\right)\right) \\
& \quad \leq \Lambda\left(H\left(\left[\widehat{S}\left(r_{p(n)}\right]_{\alpha_{\hat{S}}\left(r_{p(n)}\right)},\left[\Gamma(r)_{q(n)}\right]_{\alpha_{\Gamma}\left(r_{q(n)}\right)}\right)\right)\right. \\
& \quad \leq Y\left(\Lambda\left(M\left(r_{p(n)}, r_{q(n)}\right)\right)\right)+L E\left(r_{p(n)}, r_{q(n)}\right),
\end{aligned}
$$

where

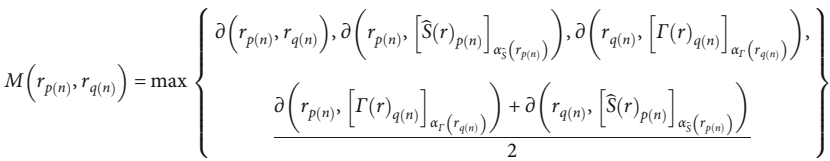

$$
\begin{aligned}
& \leq \max \left\{\begin{array}{c}
\partial\left(r_{p(n)}, r_{q(n)}\right), \partial\left(r_{p(n)}, r_{p(n)+1}\right), \partial\left(r_{q(n)}, r_{q(n)+1}\right) \\
\frac{\partial\left(r_{p(n)}, r_{q(n)+1}\right)+\partial\left(r_{q(n)}, r_{p(n)+1}\right)}{2}
\end{array}\right\},
\end{aligned}
$$

$$
\begin{aligned}
E\left(r_{p(n)}, r_{q(n)}\right) & =\min \left\{\begin{array}{l}
\partial\left(r_{p(n)},\left[\hat{S}(r)_{p(n)}\right]_{\alpha_{S}\left(r_{p(n)}\right)}\right), \partial\left(r_{q(n)},\left[\Gamma(r)_{q(n)}\right]_{\alpha_{F}\left(r_{q(n)}\right)}\right), \\
\partial\left(r_{p(n)},\left[\Gamma(r)_{q(n)}\right]_{\alpha_{r}\left(r_{q(n)}\right)}\right), \partial\left(r_{q(n)},\left[\widehat{S}(r)_{p(n)}\right]_{\alpha_{\tilde{S}}\left(r_{p(n)}\right)}\right)
\end{array}\right\} \\
& \leq \min \left\{\begin{array}{c}
\partial\left(r_{p(n)}, r_{p(n)+1}\right), \partial\left(r_{q(n)}, r_{q(n)+1}\right), \\
\partial\left(r_{p(n)}, r_{q(n)+1}\right), \partial\left(r_{q(n)}, r_{p(n)+1}\right)
\end{array}\right\}=0 .
\end{aligned}
$$

Letting $n \longrightarrow \infty$ in the above inequality, since $Y$ and $\Lambda$ are continuous and by using (48), (49), (51), and (54), we get

$$
\begin{aligned}
\Lambda(\varepsilon) & =\lim _{n \rightarrow \infty} \Lambda\left(\partial\left(r_{p(n)+1}, r_{q(n)+1}\right)\right) \\
& \leq \lim _{n \rightarrow \infty} Y\left(\Lambda\left(M\left(r_{p(n)}, r_{q(n)}\right)\right)\right) \\
& =Y(\Lambda(\varepsilon))<\Lambda(\varepsilon) .
\end{aligned}
$$


It is a contradiction, so $\left\{r_{n}\right\}$ is Cauchy. Since $\omega$ is complete, $\left\{r_{n}\right\}$ converges to $r^{*} \in \omega$, i.e., $\lim _{n \rightarrow \infty} d\left(r_{n}, r^{*}\right)=0$. We claim that $r^{*} \in\left[\Gamma\left(r^{*}\right)\right]_{\alpha_{T}\left(x^{*}\right)}$. Assume that $r^{*} \notin$ $\left[\Gamma\left(r^{*}\right)\right]_{\alpha_{T}\left(x^{*}\right)}$ (that is, $\left.\partial\left(r^{*},\left[\Gamma\left(r^{*}\right)\right]_{\alpha_{T}\left(r^{*}\right)}\right)>0\right)$, then there are $n_{0} \in \mathbb{N}$ and a subsequence $\left\{r_{n_{k}}\right\}$ of $\left\{r_{n}\right\}$ so that $\partial\left(r_{2 n_{k}+1}\right.$, $\left.\left[\Gamma\left(r^{*}\right)\right]_{\alpha_{\Gamma}\left(r^{*}\right)}\right)>0$, for all $n_{k} \geq n_{0}$. Since $\partial\left(r_{2 n_{k}+1},\left[\Gamma\left(r^{*}\right)\right]_{\alpha_{\Gamma}\left(r^{*}\right)}\right)$ $>0$, for all $n_{k} \geq n_{0}$, so by $(\Phi 1)$, we have

$$
\begin{aligned}
& \Lambda\left(\partial\left(r_{2 n_{k}+1},\left[\Gamma\left(r^{*}\right)\right]_{\alpha_{\Gamma}\left(r^{*}\right)}\right)\right) \\
& \quad \leq \Lambda\left(H\left(\left[\widehat{S}\left(r_{2 n_{k}}\right)\right]_{\alpha_{\widehat{S}}\left(r_{2 n_{k}}\right)},\left[\Gamma\left(r^{*}\right)\right]_{\alpha_{\Gamma}\left(r^{*}\right)}\right)\right) \\
& \quad \leq Y\left(\Lambda\left(M\left(r_{2 n_{k}}, r^{*}\right)\right)\right)+L E\left(r_{2 n_{k}}, r^{*}\right),
\end{aligned}
$$

where

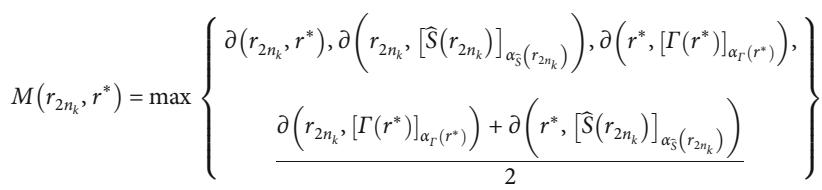

$$
\begin{aligned}
& \leq \max \left\{\begin{array}{c}
\partial\left(r_{2 n_{k}}, r^{*}\right), \partial\left(r_{2 n_{k}}, r_{2 n_{k}+1}\right), \partial\left(r^{*},\left[\Gamma\left(r^{*}\right)\right]_{\alpha_{r}\left(r^{*}\right)}\right), \\
\frac{\partial\left(r_{2 n_{k}},\left[\Gamma\left(r^{*}\right)\right]_{\alpha_{r}\left(r^{*}\right)}\right)+\partial\left(r^{*}, r_{2 n_{k}+1}\right)}{2}
\end{array}\right\}, \\
& E\left(r_{2 n_{k}}, r^{*}\right)=\min \left\{\begin{array}{l}
\partial\left(r_{2 n_{k^{\prime}}},\left[\widehat{S}\left(r_{2 n_{k}}\right)\right]_{\alpha_{\hat{S}}\left(r_{2 n_{k}}\right)}\right), \partial\left(r^{*},\left[\Gamma\left(r^{*}\right)\right]_{\alpha_{\Gamma}\left(r^{*}\right)}\right), \\
\partial\left(r_{2 n_{n^{\prime}}},\left[\Gamma\left(r^{*}\right)\right]_{\alpha_{\Gamma}\left(r^{*}\right)}\right), \partial\left(r^{*},\left[\widehat{S}\left(r_{2 n_{k}}\right)\right]_{\alpha_{\hat{S}}\left(r_{2 n_{k}}\right)}\right)
\end{array}\right\} \\
& \leq \min \left\{\begin{array}{l}
\partial\left(r_{2 n_{k}}, r_{2 n_{k}+1}\right), \partial\left(r^{*},\left[\Gamma\left(r^{*}\right)\right]_{\alpha_{\Gamma}\left(r^{*}\right)}\right), \\
\partial\left(r_{2 n_{k}},\left[\Gamma\left(r^{*}\right)\right]_{\alpha_{\Gamma}\left(r^{*}\right)}\right), \partial\left(r^{*}, r_{2 n_{k}+1}\right)
\end{array}\right\} .
\end{aligned}
$$
have

Letting $k \longrightarrow \infty$ and using the continuity of $\Lambda$ and $Y$, we

$$
\begin{aligned}
\Lambda\left(\partial\left(r^{*},\left[\Gamma\left(r^{*}\right)\right]_{\alpha_{\Gamma}\left(r^{*}\right)}\right)\right) \leq & Y\left(\Lambda\left(\partial\left(r^{*},\left[\Gamma\left(r^{*}\right)\right]_{\alpha_{\Gamma}\left(r^{*}\right)}\right)\right)\right) \\
& +0<\Lambda\left(\partial\left(r^{*},\left[\Gamma\left(r^{*}\right)\right]_{\alpha_{\Gamma}\left(r^{*}\right)}\right)\right),
\end{aligned}
$$

which is a contradiction. Hence, $\partial\left(r^{*},\left[\Gamma\left(r^{*}\right)\right]_{\alpha_{\Gamma}\left(r^{*}\right)}\right)=0$, and $r^{*} \in\left[\Gamma\left(r^{*}\right)\right]_{\alpha_{\Gamma}\left(r^{*}\right)}$. Similarly, one can easily prove that $r^{*} \in$ $\left[\widehat{S}\left(r^{*}\right)\right]_{\alpha_{\hat{S}}\left(r^{*}\right)}$. Therefore, $r^{*} \in\left[\widehat{S}\left(r^{*}\right)\right]_{\alpha_{\hat{S}}\left(r^{*}\right)} \cap\left[\Gamma\left(r^{*}\right)\right]_{\Gamma\left(r^{*}\right)}$.

Example 17. Let $\omega=[0,1]$ endowed with the metric $\partial(r, \widehat{J})$ $=|r-\widehat{J}|$. Consider $\Lambda, Y:(0, \infty) \longrightarrow(0, \infty)$ as $\Lambda(t)=t$ and $Y(t)=99 t / 100$. Here, $\Lambda, Y \in \Phi^{*}$ and $Y$ are continuous. For $\alpha \in(0,1]$, given $\widehat{S}, \Gamma: \omega \longrightarrow \Im(\omega)$ by

$$
\begin{gathered}
\widehat{S}(r)(t)= \begin{cases}\alpha, & 0 \leq t \leq \frac{r}{60} \\
\frac{\alpha}{2}, & \frac{r}{60} \leq t \leq \frac{r}{40} \\
\alpha & \frac{r}{40} \leq t \leq \frac{r}{20} \\
\frac{\alpha}{\alpha}, & \frac{r}{20} \leq t \leq 1\end{cases} \\
\Gamma(r)(t)= \begin{cases}\alpha, & 0 \leq t \leq \frac{r}{15} \\
\frac{\alpha}{3}, & \frac{r}{15} \leq t \leq \frac{r}{10} \\
\frac{\alpha}{4}, & \frac{r}{10} \leq t \leq \frac{r}{5} \\
\frac{\alpha}{7}, & \frac{r}{5} \leq t \leq 1\end{cases}
\end{gathered}
$$

such that

$$
\begin{aligned}
{[\widehat{S}(r)]_{\alpha} } & =\left[0, \frac{r}{60}\right], \\
{[\Gamma(r)]_{\alpha} } & =\left[0, \frac{r}{15}\right] .
\end{aligned}
$$

For $r, \widehat{J} \in \omega$ with $\left.H(\widehat{S}(r)]_{\alpha_{\widehat{S}}(r)},[\Gamma(\widehat{J})]_{\alpha_{\Gamma}(\widehat{J})}\right)>0$, we have

$$
\Lambda\left(H\left([\widehat{S}(r)]_{\alpha_{\widehat{S}(r)},}[\Gamma(\widehat{J})]_{\alpha_{\Gamma}(\widehat{J})}\right)\right) \leq Y(\Lambda(M(r, \widehat{J})))+L E(r, \widehat{J}) .
$$

where

$$
M(r, \widehat{J})=\max \left\{\begin{array}{c}
\partial(r, \widehat{J}), \partial\left(r,[\widehat{S}(r)]_{\alpha_{\tilde{S}}(r)}\right), \\
\partial\left(\hat{J},[\Gamma(\widehat{J})]_{\alpha_{r}(\widehat{J})}\right), \frac{\partial\left(r,[\Gamma(\widehat{J})]_{\alpha_{r}(\widehat{J})}\right)+\partial\left(\widehat{J},[\widehat{S}(r)]_{\alpha_{\widehat{S}}(r)}\right)}{2}
\end{array}\right\},
$$

$E(r, \widehat{J})=\min \left\{\begin{array}{c}\partial\left(r,[\widehat{S}(r)]_{\left.\alpha_{\widehat{S}(r)}\right)}\right), \partial\left(\widehat{J},[\Gamma(\widehat{J})]_{\alpha_{\Gamma}(\widehat{J})}\right) \\ , \partial\left(r,[\Gamma(\widehat{J})]_{\alpha_{\Gamma}(\widehat{J})}\right), \partial\left(\widehat{J},[\widehat{S}(r)]_{\alpha_{\widehat{S}}(r)}\right)\end{array}\right\}$.

Hence, all the conditions of Theorem 16 (with $L=1$ ) are satisfied, and $0 \in[S 0]_{\alpha} \cap[T 0]_{\alpha}$.

Corollary 18. Let $\widehat{S}, \Gamma$ be FM from $\omega$ into $\mathfrak{\Im}(\omega)$, and for each $r \in \omega$, there exist $\alpha_{\widehat{S}}(r), \alpha_{\Gamma}(r) \in(0,1]$ such that $[\widehat{S}(r)]_{\alpha_{\hat{S}}(r)}$ and $[\Gamma(\widehat{J})]_{\alpha_{\Gamma}(\widehat{J})}$ are nonempty, closed, and bounded subsets of $\omega$. Assume that there are $Y \in \Psi$ and $\Lambda \in \Phi$ such that for all $r, \widehat{J}$ $\in \omega$, 


$$
\begin{aligned}
& H\left([\widehat{S}(r)]_{\alpha_{\widehat{S}}(r)},[\Gamma(\widehat{J})]_{\alpha_{\Gamma}(\widehat{J})}\right) \\
& \quad>0 \Rightarrow \Lambda\left(H\left([\widehat{S}(r)]_{\alpha_{\widehat{S}(r)},},[\Gamma(\widehat{J})]_{\alpha_{T}(\widehat{J})}\right)\right) \\
& \quad \leq Y(\Lambda(M(r, \widehat{J}))),
\end{aligned}
$$

where $M(r, \widehat{J})$ is defined by (20). If $Y$ is continuous, then there is $u \in \omega$ such that $u \in[\widehat{S}(u)]_{\alpha_{\widehat{S}}(u)} \cap[\Gamma(u)]_{\alpha_{\Gamma}(u)}$.

Proof. Set $L=0$ in Theorem 16 .

Corollary 19. Let $\widehat{S}$ be a $F M$ from $\omega$ into $\mathfrak{\Im}(\omega)$, and for each $r \in \omega$, there exist $\alpha_{\widehat{S}}(r) \in(0,1]$ such that $[\widehat{S}(r)]_{\alpha_{\hat{S}}(r)}$ is nonempty, closed, and bounded subsets of $\omega$. Assume that there are $Y \in \Psi, \Lambda \in \Phi$, and $L \geq 0$ such that for all $r, \widehat{J} \in \omega$,

$$
\begin{aligned}
& H\left([\widehat{S}(r)]_{\alpha_{\hat{S}}(r)},[\widehat{S}(\widehat{J})]_{\alpha_{\widehat{S}}(\widehat{J})}\right) \\
& \quad>0 \Rightarrow \Lambda\left(H\left([\widehat{S}(r)]_{\alpha_{\widehat{S}}(r)},[\widehat{S}(\widehat{J})]_{\alpha_{\widehat{S}}(\widehat{J})}\right)\right) \\
& \quad \leq Y(\Lambda(M(r, \widehat{J})))+L E(r, \widehat{J}),
\end{aligned}
$$

where

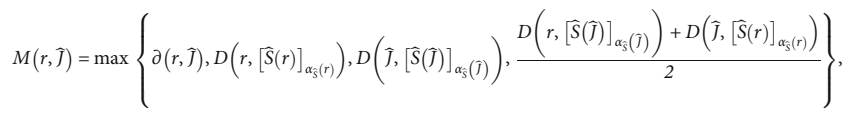

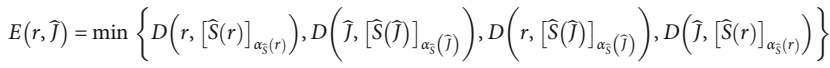

If $Y$ is continuous, then there is $u \in \omega$ so that $u \in$ $[\widehat{S}(u)]_{\alpha_{\hat{S}}(u)}$.

Proof. Take $\widehat{S}=\Gamma$ in Theorem 16 .

Now, we consider multivalued mappings.

Theorem 20. Let $F, G: \omega \longrightarrow C B(\omega)$ be multivalued mappings. Assume that there are $Y \in \Psi, \Lambda \in \Phi$, and $L \geq 0$ such that for all $r, \widehat{J} \in \omega$,

$$
\begin{aligned}
H(F(r), G(\widehat{J})) & >0 \Rightarrow \Lambda(H(F(r), G(\widehat{J}))) \\
& \leq Y(\Lambda(M(r, \widehat{J})))+L E(r, \widehat{J}),
\end{aligned}
$$

where

$$
\begin{gathered}
M(r, \widehat{J})=\max \left\{\partial(r, \widehat{J}), \partial(r, F(r)), \partial(\widehat{J}, G(\widehat{J})), \frac{\partial(r, G(\widehat{J}))+\partial(\widehat{J}, F(r))}{2}\right\}, \\
E(r, \widehat{J})=\min \{\partial(r, F(r)), \partial(\widehat{J}, G(\widehat{J})), \partial(r, G(\widehat{J})), \partial(\widehat{J}, F(r))\} .
\end{gathered}
$$

If $Y$ is continuous, then there is $u \in \omega$ such that $u \in F(u)$ $\cap G(u)$.

Proof. Consider a mapping $\alpha: \omega \longrightarrow(0,1]$ and a pair of fuzzy mappings $\widehat{S}, \Gamma: \omega \longrightarrow \sqsubseteq(\omega)$ defined by

$\widehat{S}(r)(t)=\left\{\begin{array}{ll}\alpha(r), & \text { if } t \in F(r) \\ 0, & \text { if } t \notin F(r),\end{array}\right.$ and $\Gamma(r)(t)= \begin{cases}\alpha(r), & \text { if } t \in G(r) \\ 0, & \text { if } t \notin G(r) .\end{cases}$

Then,

$$
\begin{gathered}
{[\widehat{S}(r)]_{\alpha(r)}=\{t: \widehat{S}(r)(t) \geq \alpha(r)\}=F(r),} \\
{[\Gamma(r)]_{\alpha(r)}=\{t: \Gamma(r)(t) \geq \alpha(r)\}=G(r) .}
\end{gathered}
$$

Thus, by Theorem 16, there is $u \in \omega$ so that $u \in$ $\widehat{\widehat{S}}(u)]_{\alpha_{\widehat{S}}(u)} \cap[\Gamma(u)]_{\alpha_{\Gamma}(u)}=F(u) \cap G(u)$.

Theorem 21. Let $\widehat{S}, \Gamma: \omega \longrightarrow W(\omega)$ be FM. Assume that there are $Y \in \Psi, \Lambda \in \Phi$, and $L \geq 0$ such that for all $r, \widehat{J} \in \omega$,

$$
\begin{aligned}
\partial_{\infty}(\widehat{S}(r), \Gamma(\widehat{J})) & >0 \Rightarrow \Lambda\left(\partial_{\infty}(\widehat{S}(r), \Gamma(\widehat{J}))\right) \\
& \leq Y(\Lambda(M(r, \widehat{J})))+L E(r, \widehat{J}),
\end{aligned}
$$

where

$$
M(r, \widehat{J})=\max \left\{p(r, \widehat{J}), p(r, \widehat{S}(r)), p(\widehat{J}, \Gamma(\widehat{J})), \frac{p(r, \Gamma(\widehat{J}))+p(\widehat{J}, \widehat{S}(r))}{2}\right\},
$$

$E(r, \widehat{J})=\min \{p(r, \widehat{S}(r)), p(\widehat{J}, \Gamma(\widehat{J})), p(r, \Gamma(\widehat{J})), p(\widehat{J}, \widehat{S}(r))\}$

If $Y$ is continuous, then there is $u \in \omega$ so that $\{u\} \subset \widehat{S}(u)$ and $\{u\} \subset \Gamma(u)$.

Proof. Let $r \in \omega$; then by Lemma 14 , there is $\widehat{J} \in \omega$ so that $\widehat{J}$ $\in[\widehat{S}(r)]_{1}$. Similarly, we can find $e \in \omega$ such that $e \in[\Gamma(r)]_{1}$. It follows that for each $r \in \omega,[\widehat{S}(r)]_{\alpha(r)}$ and $[\Gamma(r)]_{\alpha(r)}$ are nonempty, closed, and bounded subsets of $\omega$. As $\alpha(r)=\alpha(\widehat{J})=1$, by the definition of a $\partial_{\infty}$-metric for fuzzy sets, we have

$$
H\left([\widehat{S}(r)]_{\alpha(r)},[\Gamma(\widehat{J})]_{\alpha(\widehat{J})}\right) \leq \partial_{\infty}(\widehat{S}(r), \Gamma(\widehat{J})) \text {, forall } r, \widehat{J} \in \omega .
$$

Since $\phi$ is nondecreasing, we have

$$
\begin{aligned}
& \Lambda\left(H\left([\widehat{S}(r)]_{\alpha(r)},[\Gamma(\widehat{J})]_{\alpha(\widehat{J})}\right)\right) \\
& \quad \leq \Lambda\left(\partial_{\infty}(\widehat{S}(r), \Gamma(\widehat{J}))\right) \leq Y(\Lambda(M(r, \widehat{J})))+L E(r, \widehat{J}),
\end{aligned}
$$


where

$$
M(r, \widehat{J})=\max \left\{p(r, \widehat{J}), p(r, \widehat{S}(r)), p(\widehat{J}, \Gamma(\widehat{J})), \frac{p(r, \Gamma(\widehat{J}))+p(\widehat{J}, \widehat{S}(r))}{2}\right\},
$$

$$
E(r, \widehat{J})=\min \{p(r, \widehat{S}(r)), p(\widehat{J}, \Gamma(\widehat{J})), p(r, \Gamma(\widehat{J})), p(\widehat{J}, \widehat{S}(r))\},
$$

for all $r, \widehat{J} \in \omega$. Since $[\widehat{S}(r)]_{1} \subseteq[\widehat{S}(r)]_{\alpha(r)}$, we have

$$
\partial_{\infty}\left(r,[\widehat{S}(r)]_{\alpha(r)}\right) \leq \partial\left(r,[\widehat{S}(r)]_{1}\right) \text { for each } \alpha \in(0,1] .
$$

It yields that

$$
P(r, \widehat{S}(r)) \leq \partial\left(r,[\widehat{S}(r)]_{1}\right)
$$

Similarly,

$$
p(r, \Gamma(r)) \leq \partial\left(x,[\Gamma(r)]_{1}\right) .
$$

It yields that for all $r, \widehat{J} \in \omega$,

$$
\Lambda\left(H\left([\widehat{S}(r)]_{1},[\Gamma(r)]_{1}\right)\right) \leq Y(\Lambda(M(r, \widehat{J})))+L E(r, \widehat{J}),
$$

where

$$
M(r, \widehat{J})=\max \left\{\partial(r, \widehat{J}), \partial\left(r,[\widehat{S}(r)]_{1}\right), \partial\left(\widehat{J},[\Gamma(r)]_{1}\right), \frac{\partial\left(r,[\Gamma(\widehat{J})]_{1}\right)+\partial\left(\widehat{J},[\widehat{S}(r)]_{1}\right)}{2}\right\},
$$

$E(r, \widehat{J})=\min \left\{\partial\left(r,[\widehat{S}(r)]_{1}\right), \partial\left(\widehat{J},[\Gamma(\widehat{J})]_{1}\right), \partial\left(r,[\Gamma(\widehat{J})]_{1}\right), \partial\left(\widehat{J},[\widehat{S}(r)]_{1}\right)\right\}$.

By Theorem 16, we obtain $u \in \omega$ such that $[\widehat{S}(u)]_{1} \cap$ $[\Gamma(u)]_{1}$, that is, $\{u\} \subset \widehat{S}(u)$ and $\{u\} \subset \Gamma(u)$.

Corollary 22. Let $(\phi, \partial)$ be a complete $M S$, and $\widehat{S}, \Gamma: \omega \longrightarrow$ $W(\omega)$ be FM. Assume that there exist $Y \in \Psi$ and $\Lambda \in \Phi$ such that for all $r, \widehat{J} \in \omega$,

$$
\partial_{\infty}(\widehat{S}(r), \Gamma(\widehat{J}))>0 \Rightarrow \Lambda\left(\partial_{\infty}(\widehat{S}(r), \Gamma(\widehat{J}))\right) \leq Y(\Lambda(M(r, \widehat{J}))),
$$

where $M(r, \widehat{J})$ is defined by (80). If $Y$ is continuous, then there is $u \in \omega$ so that $\{u\} \subset \widehat{S}(u)$ and $\{u\} \subset \Gamma(u)$.

Proof. Take $L=0$ in Theorem 21 .

We denote by $\widehat{S}$ (for details, see $[43,44]$ ) the setvalued mapping induced by a FM $\widehat{S}: \omega \longrightarrow F(\omega)$, i.e.,

$$
\widehat{S}(r)=\left\{\widehat{J}: \widehat{S}(\widehat{J})(t)=\max _{t \in \omega} \widehat{S}(r)(t)\right\} .
$$

Corollary 23. Let $\widehat{S}, \Gamma: \omega \longrightarrow \mathfrak{I}(\omega)$ be FM such that for all $r \in \omega, \widehat{S}(r)$ and $\widehat{\Gamma}(r)$ are nonempty, closed, and bounded subsets of $\omega$. Assume that there exist $Y \in \Psi, \Lambda \in \Phi$, and $L \geq$ 0 such that for all $r, \widehat{J} \in \omega$,

$$
\begin{aligned}
H(\widehat{S}(r), \widehat{\Gamma}(\widehat{J})) & >0 \Rightarrow \Lambda(H(\widehat{S}(r), \widehat{\Gamma}(\widehat{J}))) \\
& \leq Y(\Lambda(M(r, \widehat{J})))+L E(r, \widehat{J}),
\end{aligned}
$$

where

$$
M(r, \widehat{J})=\max \left\{\partial(r, \widehat{J}), \partial(r, \widehat{S}(r)), \partial(\widehat{J}, \widehat{\Gamma}(\widehat{J})), \frac{\partial(r, \widehat{\Gamma}(\widehat{J}))+\partial(\widehat{J}, \widehat{S}(r))}{2}\right\},
$$

$E(r, \widehat{J})=\min \{\partial(r, \widehat{S}(r)), \partial(\widehat{J}, \widehat{\Gamma}(\widehat{J})), \partial(r, \widehat{\Gamma}(\widehat{J})), \partial(\widehat{J}, \widehat{S}(r))\}$

If $Y$ is continuous, then there is $r^{*} \in \omega$ so that $\widehat{S}\left(r^{*}\right)\left(r^{*}\right)$ $\geq \widehat{S}\left(r^{*}\right)(r)$ and $\Gamma\left(r^{*}\right)\left(r^{*}\right) \geq \Gamma\left(r^{*}\right)(r)$, for all $r \in \omega$.

Proof. By Theorem 16, there is $r^{*} \in \omega$ so that $r^{*} \in \widehat{S}\left(r^{*}\right) \cap \widehat{\Gamma}$ $\left(r^{*}\right)$. Then by Lemma 15 , we have

$$
\begin{gathered}
\widehat{S}\left(r^{*}\right)\left(r^{*}\right) \geq \widehat{S}\left(r^{*}\right)(r), \\
\Gamma\left(r^{*}\right)\left(r^{*}\right) \geq \Gamma\left(r^{*}\right)(r) \text { for all } r \in \omega .
\end{gathered}
$$

Corollary 24. Let $\widehat{S}, \Gamma: \omega \longrightarrow \mathfrak{I}(\omega)$ be FM such that for all $r \in \omega, \widehat{S}(r)$ and $\widehat{\Gamma}(r)$ are nonempty, closed, and bounded subsets of $\omega$. Assume that there are $Y \in \Psi, \Lambda \in \Phi$, and $L \geq 0$ so that for all $r, \widehat{J} \in \omega$,

$H(\widehat{S}(r), \widehat{\Gamma}(\widehat{J}))>0 \Rightarrow \Lambda(H(\widehat{S}(r), \widehat{\Gamma}(\widehat{J}))) \leq Y(\Lambda(M(r, \widehat{J})))$,

where

$$
M(r, \widehat{J})=\max \left\{\partial(r, \widehat{J}), \partial(r, \widehat{S}(r)), \partial(\widehat{J}, \widehat{\Gamma}(\widehat{J})), \frac{\partial(r, \widehat{\Gamma}(\widehat{J}))+\partial(\widehat{J}, \widehat{S}(r))}{2}\right\} .
$$

If $Y$ is continuous, then there is $r^{*} \in \omega$ such that $\left(r^{*}\right)\left(r^{*}\right)$ $\geq \widehat{S}\left(r^{*}\right)(r)$ and $\Gamma\left(r^{*}\right)\left(r^{*}\right) \geq \Gamma\left(r^{*}\right)(r)$, for all $r \in \omega$.

Proof. Take $L=0$ in Corollary 23.

\section{Some Consequences}

Corollary 25. Let $\widehat{S}, \Gamma: \omega \longrightarrow \mathfrak{J}(\omega)$ be $F M$, and for each $r$ $\epsilon \omega$, there exist $\alpha_{\widehat{S}}(r), \alpha_{\Gamma}(r) \in(0,1]$ such that $[\widehat{S}(r)]_{\alpha_{\widehat{S}}(r)}$ and $[\Gamma(\widehat{J})]_{\alpha_{T}(r)}$ are nonempty, closed, and bounded subsets of $\omega$. Assume that there are $\lambda \in(0,1)$ and $L \geq 0$ such that for all $r$ ,$\widehat{J} \in \omega$, 


$$
H\left([\widehat{S}(r)]_{\alpha_{\widehat{S}(r)},},[\Gamma(\widehat{J})]_{\alpha_{\Gamma}(r)}\right) \leq \lambda M(r, \widehat{J})+L E(r, \widehat{J}),
$$

where $M(r, \widehat{J})$ and $E(r, \widehat{J})$ are defined by (20) and (21), respectively. Then, there is $u \in \omega$ such that $u \in[\widehat{S}(u)]_{\alpha_{\hat{S}}(u)} \cap$ $[\Gamma(u)]_{\alpha_{\Gamma}(u)}$.

Proof. Take $Y(t)=\lambda t$ and $\Lambda(t)=t$ in Theorem 16 .

Corollary 26. Let $\widehat{S}, \Gamma: \omega \longrightarrow \mathfrak{J}(\omega)$ be $F M$, and for each $r$ $\epsilon \omega$, there are $\alpha_{\hat{S}}(r), \alpha_{\Gamma}(r) \in(0,1]$, such that $[\widehat{S}(r)]_{\alpha_{\hat{S}}(r)}$ and $[\Gamma(\widehat{J})]_{\alpha_{\Gamma}(r)}$ are nonempty, closed, and bounded subsets of $\omega$. Assume that there are $\theta \in \Theta^{*}$ and $k \in(0,1)$ such that for all $\left.r, \widehat{J} \in \omega, H(\widehat{S}(r)]_{\alpha_{\widehat{S}}(r)},[\Gamma(\widehat{J})]_{\alpha_{T}(r)}\right)>0$ implies

$$
\theta\left(H\left([\widehat{S}(r)]_{\alpha_{\widehat{S}(r)}},[\Gamma(\widehat{J})]_{\alpha_{\Gamma}(r)}\right)\right) \leq[\theta(M(r, \widehat{J}))]^{k},
$$

where $M(r, \widehat{J})$ is defined by (20). Then, there is $u \in \omega$ such that $u \in[\widehat{S}(u)]_{\alpha_{\widehat{S}}(u)} \cap[\Gamma(u)]_{\alpha_{T}(u)}$.

Proof. Take $Y(t):=(\ln k) t, \Lambda(t)=\ln t$, and $L=0$ in Theorem 16.

Corollary 27. Let $\widehat{S}, \Gamma: \omega \longrightarrow \mathfrak{\Im}(\omega)$ be $F M$, and for each $r$ $\in \omega$, there exist $\alpha_{\widehat{S}}(r), \alpha_{\Gamma}(r) \in(0,1]$, such that $[\widehat{S}(r)]_{\alpha_{\hat{S}}(r)}$ and $[\Gamma(\widehat{J})]_{\alpha_{\Gamma}(r)}$ are nonempty, closed, and bounded subsets of $\omega$. Assume that there are $F \in \Xi^{*}$ and $\tau>0$ such that for all $r, \widehat{J}$ $\left.\in \omega, H(\widehat{S}(r)]_{\alpha_{\widehat{S}}(r)},[\Gamma(\widehat{J})]_{\alpha_{\Gamma}(r)}\right)>0$ implies

$$
\tau+F\left(H\left([\widehat{S}(r)]_{\alpha_{\widehat{S}(r)}},[\Gamma(\widehat{J})]_{\alpha_{\Gamma}(r)}\right)\right) \leq F(r, \widehat{J}),
$$

where $M(r, \widehat{J})$ is defined by (20). Then, there is $u \in X$ so that $u \in \widehat{S}(u)]_{\alpha_{\widehat{S}}(u)} \cap[\Gamma(u)]_{\alpha_{T}(u)}$.

Proof. Take $Y(t)=e^{-\tau} t, \Lambda(t)=e^{t}$, and $L=0$ in Theorem 16 .

Corollary 28. Let $\widehat{S}, \Gamma: \omega \longrightarrow \mathfrak{I}(\omega)$ be $F M$, and for each $r$ $\epsilon \omega$, there exist $\alpha_{\widehat{S}}(r), \alpha_{\Gamma}(r) \in(0,1]$, such that $[\widehat{S}(r)]_{\alpha_{\widehat{S}}(r)}$ and $[\Gamma(\widehat{J})]_{\alpha_{\Gamma}(r)}$ nonempty, closed, and bounded subsets of $\omega$. Assume that there is $L \geq 0$ such that for all $r, \widehat{J} \in \omega$ implies

$$
H\left([\widehat{S}(r)]_{\alpha_{\widehat{S}}(r)},[\Gamma(\widehat{J})]_{\alpha_{\Gamma}(r)}\right) \leq \beta(M(r, \widehat{J})) M(r, \widehat{J})+L E(r, \widehat{J}),
$$

where $M(r, \widehat{J})$ and $E(r, \widehat{J})$ are defined by (20) and (21), respectively, and $\beta:\left[0,{ }^{\prime} \infty\right) \longrightarrow[0, \infty)$ is such that $\lim _{r \rightarrow t^{+}} \beta(r)<1$ for each $t \in(0, \infty)$. Then, there is $u \in \omega$ such that $u \in[\widehat{S}(u)]_{\alpha_{\widehat{S}}(u)}$ $\cap[\Gamma(u)]_{\alpha_{T}(u)}$.

Proof. It follows from Theorem 16 by taking $Y(t):=\beta(t) t$ and $Y(t)=t$.

\section{Application to an Ordinary Fuzzy Differential Equation}

In this section, we apply our obtained results to study the existence of a solution for the second order nonlinear boundary value problem:

$$
\left\{\begin{array}{ll}
r^{\prime \prime}(t)=K\left(t, r(t), r^{\prime}(t)\right), & t \in[0, a], a>0 \\
r\left(t_{1}\right)=r_{1}, & t_{1} \in[0, a] \\
r\left(t_{2}\right)=r_{2}, & t_{2} \in[0, a]
\end{array},\right.
$$

where $K:[0, a] \times W(\omega) \times W(\omega) \longrightarrow W(\omega)$ is a continuous function. This problem is equivalent to the integral equation $([45,46,48])$ :

$$
r(t)=\int_{t_{1}}^{t_{2}} G(t, s) K\left(s, r(s), r^{\prime}(s)\right) d s+\beta(t), t \in[0, a],
$$

where Green's function $G$ is given by

$$
G(t, s)= \begin{cases}\frac{\left(t_{2}-t\right)(s-t)}{t_{2}-t_{1}} & , \text { if } t_{1} \leq s \leq t \leq t_{2}, \\ \frac{\left(t_{2}-s\right)\left(t-t_{1}\right)}{t_{2}-t_{1}} & , \text { if } t_{1} \leq t \leq s \leq t_{2}\end{cases}
$$

and $\beta(t)$ satisfies $\beta^{\prime \prime}=0, \beta\left(t_{1}\right)=r_{1}$, and $\beta\left(t_{2}\right)=r_{2}$. Let us recall some properties of $G(t, s)$. Particularly,

$$
\begin{gathered}
\int_{t_{1}}^{t_{2}}|G(t, s)| d s \leq \frac{\left(t_{2}-t_{1}\right)^{2}}{8}, \\
\int_{t_{1}}^{t_{2}}\left|G_{t}(t, s)\right| d s \leq \frac{t_{2}-t_{1}}{2} .
\end{gathered}
$$

Our investigation is based on the existence of a common fixed point for a pair of integral operators given as follows:

$$
\widehat{S}(r)(t)=\int_{t_{1}}^{t_{2}} G(t, s) K_{1}\left(s, r(s), r^{\prime}(s)\right) d s+\beta(t), t \in[0, a],
$$

$$
\Gamma(r)(t)=\int_{t_{1}}^{t_{2}} G(t, s) K_{2}\left(s, r(s), r^{\prime}(s)\right) d s+\beta(t), t \in[0, a]
$$

where $K_{1}, K_{2} \in C([0, a] \times W(\omega) \times W(\omega), W(\omega)), x \in C^{1}([0$, $a], W(\omega))$, and $\beta \in C([0, a], W(\omega))$.

\section{Theorem 29. Suppose that}

(a) $K_{1}, K_{2}:[0, a] \times W(\omega) \times W(\omega) \longrightarrow W(\omega)$ are increasing in their second and third variables, 
(b) There is $r_{0} \in C^{1}([0, a], W(\omega))$ such that for all $t \in[0$, a], we have

$$
r_{0}(t)=\int_{t_{1}}^{t_{2}} G(t, s) K_{1}\left(s, r_{0}(s), r_{0}^{\prime}(s)\right) d s+\beta(t), t_{1}, t_{2} \in[0, a],
$$

(c) There are $\gamma, \delta>0$ such that for all $t \in[0, a]$, we have

$$
\begin{gathered}
\left|K_{1}\left(t, r(t), r^{\prime}(t)\right)-K_{2}\left(t, \widehat{J}(t), \widehat{J}^{\prime}(t)\right)\right| \\
\quad \leq \gamma|r(t)-\widehat{J}(t)|+\delta\left|r^{\prime}(t)-\widehat{J}^{\prime}(t)\right|,
\end{gathered}
$$

for all $r, \widehat{J} \in C^{1}([0, a], W(\omega))$, with $K_{1}(., .,.) \neq K_{2}$ $(., .,$.$) ,$

(d) For $\gamma, \delta>0$ and $t_{1}, t_{2} \in[0, a]$, we have

$$
\gamma \frac{\left(t_{2}-t_{1}\right)^{2}}{8}+\delta \frac{\left(t_{2}-t_{1}\right)}{2}<\frac{9}{10},
$$

(e) if $r, \widehat{J} \in C^{l}([0, a], W(\omega))$ is comparable, then every $u$ $\in\left(\Gamma_{1} r\right)_{1}$ and every $v \in\left(\Gamma_{1} \widehat{J}\right){ }_{1}$ are comparable.

Then, the pair of nonlinear integral equations

$$
\begin{aligned}
& r(t)=\int_{t_{1}}^{t_{2}} G(t, s) K_{1}\left(s, r(s), r^{\prime}(s)\right) d s+\beta(t), t \in[0, a], \\
& r(t)=\int_{t_{1}}^{t_{2}} G(t, s) K_{2}\left(s, r(s), r^{\prime}(s)\right) d s+\beta(t), t \in[0, a],
\end{aligned}
$$

has a common solution in $C^{1}\left(\left[t_{1}, t_{2}\right], W(\omega)\right)$.

Proof. Consider $C=C^{1}\left(\left[t_{1}, t_{2}\right], W(\omega)\right)$ with the metric

$$
\partial_{\infty}(r, \widehat{J})=\max _{t_{1} \leq t \leq t_{2}}\left\{\gamma|r(t)-\widehat{J}(t)|+\delta\left|r^{\prime}(t)-\widehat{J}^{\prime}(t)\right|\right\} .
$$

Note that $\left(C, \partial_{\infty}\right)$ is a complete linear MS. Let $\widehat{S}, \Gamma$ $: C \longrightarrow C$ be two integral operators defined by (108) and (109). Clearly, $\widehat{S}$ and $\Gamma$ are well defined since $K_{1}, K_{2}$ and $\beta$ are continuous functions. Now, $r^{*}$ is a solution of (113) and (114) if and only if $r^{*}$ is a common fixed point of $\widehat{S}$ and $\Gamma$. By hypothesis (a), $\widehat{S}, \Gamma$ is increasing. Next, for all $r, \widehat{J} \in C$ with $K_{1}(., .,.) \neq K_{2}(., .,$.$) , by hypothesis (c), we$ have successively

$$
\begin{gathered}
|\widehat{S}(r)(t)-\Gamma(\widehat{J})(t)| \leq \int_{t_{1}}^{t_{2}}|G(t, s)|\left|K_{1}\left(s, r(s), r^{\prime}(s)\right)-K_{2}\left(s, \widehat{J}(s), J \wedge^{\prime}(s)\right)\right| \\
d s \leq \partial_{\infty}(r, \widehat{J}) \int_{t_{1}}^{t_{2}}|G(t, s)| d s \leq \frac{\left(t_{2}-t_{1}\right)^{2}}{8} \partial_{\infty}(r, \widehat{J}), \\
\left|(\widehat{S}(r))^{\prime}(t)-(\Gamma(\widehat{J}))^{\prime}(t)\right| \leq \int_{t_{1}}^{t_{2}}\left|G_{t}(t, s)\right|\left|K_{1}\left(s, r(s), r^{\prime}(s)\right)-K_{2}\left(s, \widehat{J}(s), y^{\prime}(s)\right)\right| \\
d s \leq \partial_{\infty}(r, \widehat{J}) \int_{t_{1}}^{t_{2}}\left|G_{t}(t, s)\right| d s \leq \frac{\left(t_{2}-t_{1}\right)}{2} \partial_{\infty}(r, \widehat{J}) .
\end{gathered}
$$

From (116) and (117), we easily obtain

$$
\begin{aligned}
\partial_{\infty}(\widehat{S}(r), \Gamma(\widehat{J})) & \leq\left(\gamma \frac{\left(t_{2}-t_{1}\right)^{2}}{8}+\delta \frac{\left(t_{2}-t_{1}\right)}{2}\right) \partial_{\infty}(r, \widehat{J}) \\
& <\frac{9}{10} \partial_{\infty}(r, \widehat{J}) .
\end{aligned}
$$

It implies that

$$
e^{\partial_{\infty}(S \wedge(r), \Gamma(\widehat{J}))}<e^{9 / 10 \partial_{\infty}(r, \widehat{J})}<e^{\partial_{\infty}(r, \widehat{J})} .
$$

Therefore,

$$
\partial_{\infty}(\widehat{S}(r), \Gamma(\widehat{J})) e^{\partial_{\infty}(\widehat{S}(r), \Gamma(\widehat{J}))}<\frac{9}{10} \partial_{\infty}(r, \widehat{J}) e^{\partial_{\infty}(r, \widehat{J})} .
$$

Let $\Lambda, Y:(0, \infty) \longrightarrow(0, \infty)$ be defined by

$$
\begin{aligned}
& \Lambda(t)=t e^{t}, t>0, \\
& Y(t)=\frac{9 t}{10}, t>0,
\end{aligned}
$$

respectively.

Thus, we have

$$
\Lambda\left(\partial_{\infty}(\widehat{S}(r), \Gamma(\widehat{J}))\right) \leq Y\left(\Lambda\left(\partial_{\infty}(r, \widehat{J})\right)\right) \leq Y(\Lambda(M(r, \widehat{J}))) .
$$

Therefore, by Corollary 28, $\widehat{S}$ and $\Gamma$ have a common fixed point $r^{*} \in C$, that is, $r^{*}$ is a common solution of (113) and (114). As an immediate consequence of Theorem 29, in the case of $\widehat{S}=\Gamma$, we find that the integral equation (105) has a solution in $C$, and hence, the second order nonlinear boundary value problem (104) has a solution.

\section{Conclusion}

In the present work, we introduced a new concept of fuzzy mappings in complete metric spaces. Also, we derived the existence of $\alpha$-fuzzy common fixed points for two fuzzy mappings under generalized almost $(Y, \Lambda)$-contractions in complete metric spaces. We also gave an illustrative example to support our main results. We further showed some relations between multivalued mappings and fuzzy mappings, which can be utilized to ensure the existence of a common fixed point for multivalued mappings. Finally, we applied our main results to provide a solution for a second order nonlinear boundary value problem.

\section{Data Availability}

The data used to support the findings of this study are available from the corresponding author upon request. 


\section{Conflicts of Interest}

The authors declare no conflict of interest.

\section{Authors' Contributions}

All authors contributed equally in writing this article. All authors read and approved the final manuscript.

\section{References}

[1] S. Banach, "Sur les opérations dans les ensembles abstraits et leur application aux équations intégrales," Fundamenta Mathematicae, vol. 3, pp. 133-181, 1922.

[2] V. Berinde, Generalized Contractions and Applications, Editura Cub Press 22, Baia Mare, 1997.

[3] H. Aydi, E. Karapinar, and W. Shatanawi, "Coupled fixed point results for $(\psi, \varphi)$-weakly contractive condition in ordered partial metric spaces," Computers \& Mathematics with Applications, vol. 62, no. 12, pp. 4449-4460, 2011.

[4] M. Jleli and B. Samet, "A new generalization of the Banach contraction principle," Journal of Inequalities and Applications, vol. 2014, no. 1, 2014.

[5] H. Aydi, E. Karapinar, and A. F. R. L. de Hierro, "W-interpolative Ciric-Reich-Rus type contractions," Mathematics, vol. 7, no. 1, p. 57, 2019.

[6] M. Jleli, E. Karapinar, and B. Samet, "Further generalizations of the Banach contraction principle," Journal of Inequalities and Applications, vol. 2014, no. 1, 2014.

[7] H. Aydi, C. M. Chen, and E. Karapinar, "Interpolative ĆirićReich-Rus type contractions via the Branciari distance," Mathematics, vol. 7, no. 1, p. 84, 2019.

[8] F. Vetro, "A generalization of Nadler fixed point theorem," Carpathian Journal of Mathematics, vol. 31, pp. 403-410, 2015.

[9] X. D. Liu, S. S. Chang, Y. Xiiao, and L. C. Zhao, "Some fixed point theorems concerning $(\psi, \phi)$-type contraction in complete metric spaces," Journal of Nonlinear Science and Applications, vol. 9, pp. 4127-4136, 2016.

[10] H. Aydi, H. Lakzian, Z. D. Mitrovic, and S. Radenovic, "Best proximity points of MT-Cyclic contractions with property UC," Numerical Functional Analysis and Optimization, vol. 41, no. 7, pp. 871-882, 2020.

[11] E. Karapinar, S. Czerwik, and H. Aydi, “ $(\alpha, \psi)$-Meir-Keeler contraction mappings in generalized b-metric spaces," Journal of Function Spaces, vol. 2018, Article ID 3264620, 4 pages, 2018.

[12] H. A. Hançer, G. Minak, and I. Altun, "On a broad category of multivalued weakly Picard operators," Fixed Point Theory, vol. 18, no. 1, pp. 229-236, 2017.

[13] N. Mizoguchi and W. Takahashi, "Fixed point theorems for multivalued mappings on complete metric spaces," Journal of Mathematical Analysis and Applications, vol. 141, no. 1, pp. 177-188, 1989.

[14] T. Abdeljawad, N. Mlaiki, H. Aydi, and N. Souayah, "Double controlled metric type spaces and some fixed point results," Mathematics, vol. 6, no. 12, p. 320, 2018.

[15] V. Parvaneh, M. R. Haddadi, and H. Aydi, "On best proximity point results for some type of mappings," Journal of Function Spaces, vol. 2020, Article ID 6298138, 6 pages, 2020.
[16] S. B. Nadler, "Multivalued contraction mappings," Pacific Journal of Mathematics, vol. 30, no. 2, pp. 475-488, 1969.

[17] V. Berinde, "Approximating fixed points of weak contractions using the Picard iteration," Nonlinear Anal. Forum, vol. 9, pp. 43-53, 2004.

[18] V. Berinde, "General constructive fixed point theorem for Ciric-type almost contractions in metric spaces," Carpathian Journal of Mathematics, vol. 24, pp. 10-11, 2008.

[19] N. Alamgir, Q. Kiran, H. Isik, and H. Aydi, "Fixed point results via a Hausdorff controlled type metric," Advances in Difference Equations, vol. 2020, no. 1, 2020.

[20] H. Aydi, A. Felhi, E. Karapinar, and S. Sahmim, "A Nadlertype fixed point theorem in dislocated spaces and applications," Miskolc Mathematical Notes, vol. 19, no. 1, pp. 111124, 2018.

[21] P. Patle, D. Patel, H. Aydi, and S. Radenovic, "ON H+Type multivalued contractions and applications in symmetric and probabilistic spaces," Mathematics, vol. 7, no. 2, p. 144, 2019.

[22] I. Altun, G. Minak, and H. Dag, "Multivalued F-contractions on complete metric spaces," Journal of Nonlinear and Convex Analysis, vol. 16, pp. 659-666, 2015.

[23] H. Afshari, H. Aydi, and E. Karapinar, "On generalized $\alpha-\psi$ -Geraghty contractions on b-metric spaces," Georgian Mathematical Journal, vol. 27, no. 1, pp. 9-21, 2020.

[24] H. Piri and S. Rahrovi, "Generalized multivalued F-weak contractions on complete metric spaces," Sahand Communications in Mathematical Analysis, vol. 2, no. 2, pp. 1-11, 2015.

[25] E. Ameer, H. Aydi, M. Arshad, and M. De la Sen, "Hybrid Ćirić type graphic $(\Upsilon, \Lambda)$-contraction mappings with applications to electric circuit and fractional differential equations," Symmetry, vol. 12, no. 3, p. 467, 2020.

[26] E. Ameer, H. Aydi, M. Arshad, H. Alsamir, and M. Noorani, "Hybrid multivalued type contraction mappings in $\alpha \mathrm{K}$-complete partial b-metric spaces and applications," Symmetry, vol. 11, no. 1, p. 86, 2019.

[27] T. Suzuki, "A new type of fixed point theorem in metric spaces," Nonlinear Analysis, vol. 71, no. 11, pp. 5313-5317, 2009.

[28] D. Wardowski, "Fixed points of a new type of contractive mappings in complete metric spaces," Fixed Point Theory and Applications, vol. 2012, no. 1, 2012.

[29] S. Heilpern, "Fuzzy mappings and fixed point theorem," Journal of Mathematical Analysis and Applications, vol. 83, no. 2, pp. 566-569, 1981.

[30] S. C. Arora and V. Sharma, "Fixed point theorems for fuzzy mappings," Fuzzy Sets and Systems, vol. 110, pp. 127-130, 2000.

[31] Z. Aslam, J. Ahmad, and N. Sultana, "New common fixed point theorems for cyclic compatible contractions," Journal of Mathematical Analysis and Applications, vol. 8, pp. 1-12, 2017.

[32] A. Azam, M. Arshad, and P. Vetro, "On a pair of fuzzy -contractive mappings," Mathematical and Computer Modelling, vol. 52, pp. 207-214, 2010.

[33] J. Ahmad, H. Aydi, and N. Mlaiki, "Fuzzy fixed points of fuzzy mappings via F-contractions and an application," Journal of Intelligent Fuzzy Systems, vol. 37, no. 4, pp. 5487-5493, 2019.

[34] A. Asif, H. Aydi, M. Arshad, and Z. Ali, "A Novel picture Fuzzyn-Banach space with some new contractive conditions and their fixed point results," Journal of Function Spaces, vol. 2020, Article ID 6305856, 12 pages, 2020. 
[35] A. Azam and I. Beg, "Common fixed points of fuzzy maps," Mathematical and Computer Modelling, vol. 49, no. 7-8, pp. 1331-1336, 2009.

[36] R. K. Bose and D. Sahani, "Fuzzy mappings and fixed point theorems," Fuzzy Sets and Systems, vol. 21, no. 1, pp. 53-58, 1987.

[37] S. S. Chang, Y. J. Cho, B. S. Lee, J. S. Jung, and S. M. Kang, "Coincidence point theorems and minimization theorems in fuzzy metric spaces," Fuzzy Sets and Systems, vol. 88, no. 1, pp. 119-127, 1997.

[38] Y. J. Cho and N. Petrot, "Existence theorems for fixed fuzzy points with closed $\alpha$-CUT sets in complete metric spaces," Communications of the Korean Mathematical Society, vol. 26, no. 1, pp. 115-124, 2011.

[39] H. Adibi, Y. J. Cho, D. O'Regan, and R. Saadati, "Common fixed point theorems in -fuzzy metric spaces," Applied Mathematics and Computation, vol. 182, no. 1, pp. 820-828, 2006.

[40] A. Al-Rawashdeh and J. Ahmad, "Common fixed point theorems for JS-contractions," Bulletin of Mathematical Analysis and Applications, vol. 8, pp. 12-22, 2016.

[41] D. Qiu and L. Shu, "Supremum metric on the space of fuzzy sets and common fixed point theorems for fuzzy mappings," Information Sciences, vol. 178, no. 18, pp. 3595-3604, 2008.

[42] R. A. Rashwan and M. A. Ahmed, "Common fixed point theorems for fuzzy mappings," Archiv der Mathematik, vol. 38, pp. 219-226, 2002.

[43] R. Saadati, S. M. Vaezpour, and Y. J. Cho, "Quicksort algorithm: application of a fixed point theorem in intuitionistic fuzzy quasi-metric spaces at a domain of words," Journal of Computational and Applied Mathematics, vol. 228, no. 1, pp. 219-225, 2009.

[44] Z. Shi-sheng, "Fixed point theorems for fuzzy mappings (II)," Applied Mathematics and Mechanics, vol. 7, no. 2, pp. 147152, 1986.

[45] V. Lakshmikantham and R. Mohapatra, Theory of Fuzzy Differential Equations and Inclusions, Taylor \& Francis, London, 2003.

[46] M. L. Puri and D. A. Ralescu, "Fuzzy random variables," Journal of Mathematical Analysis and Applications, vol. 114, no. 2, pp. 409-422, 1986.

[47] H. M. Abu-Donia, "Common fixed point theorems for fuzzy mappings in metric space under $\phi$-contraction condition," Chaos Solitons Fractals, vol. 34, pp. 538-543, 2007.

[48] A. Hussain, "Fractional convex type contraction with solution of fractional differential equation," AIMS Mathematics, vol. 5, no. 5, pp. 5364-5380, 2020. 